\title{
Fourth-derivative relativistic quantum gravity
}

\author{
S.A. Larin ${ }^{1, *}$ \\ ${ }^{1}$ Institute for Nuclear Research of the Russian Academy of Sciences, 60th October Anniversary \\ Prospect 7a, Moscow 117312, Russia
}

\begin{abstract}
We consider relativistic quantum gravity with the action including terms quadratic in the curvature tensor. This model is known to be renormalizable. We demonstrate that the model is also unitary. New expressions for the corresponding Lagrangian and the graviton propagator within dimensional regularization are derived. We argue that the considered model is the proper candidate for the fundamental quantum theory of gravitation.
\end{abstract}

\section{Introduction}

Creation of the fundamental quantum theory of gravitation is one of the most important tasks, if not the most important task, of modern theoretical physics. It is known that the problem arises because of non-renormalizability [1] of General Relativity by Einstein.

In 1977 Stelle has proved renormalizability of the Lorentz invariant gravitational actions which include besides the Einstein-Hilbert term also terms with fourth derivatives of the metric [2]. His proof used the specific gauge where the structure of ultraviolet divergences is particularly simple. For more general gauges he made the hypothesis of the cohomological structure of divergences. Recently this hypothesis was proved for the general class of background gauges [4]. Thus we consider the fact of renormalizability of gravity with fourth derivatives of the metric, which we will call also as quadratic quantum gravity, to be well established.

But Stelle also made the statement [2,3] that quantum gravity with fourth derivatives is unphysical because it violates either unitarity or causality and the model can serve only as an effective theory in some domain of energies. Since then this model is considered to be unphysical.

In the present contribution we demonstrate that quadratic quantum gravity is in fact unitary. Hence we argue that fourth derivative gravity is the proper candidate for the fundamental quantum theory of gravitation. New expressions for the Lagrangian and for the graviton propagator of quadratic quantum gravity within dimensional regularization are derived.

\section{The main part}

Let us consider the invariant under the gauge transformations action with all possible terms quadratic in the curvature tensor

$$
S_{s y m}=\int d^{D} x \mu^{-2 \epsilon} \sqrt{-g}\left(-M_{P l}^{2} R+\alpha R_{\mu \nu} R^{\mu \nu}+\beta R^{2}+\delta R_{\mu \nu \rho \sigma} R^{\mu \nu \rho \sigma}\right),
$$

*e-mail: larin@inr.ac.ru 
where the first term is the Einstein-Hilbert action. The $\Lambda$-term is omitted in the action since we will work within peturbation theory. Here $M_{P l}^{2}=1 /(16 \pi G)$ is the squared Planck mass, $R_{\mu \nu \rho \sigma}$ is the Riemann tensor, $R_{\mu \nu}$ is the Ricci tensor, $R$ is the Ricci scalar, $\alpha, \beta$ and $\delta$ are the dimensionless coupling constants of the Lagrangian, $D=4-2 \epsilon$ is the space time dimension within dimensional regularization, $\mu$ is the parameter of dimensional regularization.

We would like to stress that dimensional regularization is presently the only practically available regularization to regularize ultraviolet divergencies preserving gauge invariance in quantum gravity.

Usually the last term in the action (1) is missed in the literature $[2,4,5]$ because of the Gauss-Bonnet topological identity

$$
\int d^{4} x \sqrt{-g}\left(R_{\mu \nu \rho \sigma} R^{\mu \nu \rho \sigma}-4 R_{\mu v} R^{\mu \nu}+R^{2}\right)=0
$$

which is valid for space-times topologically equivalent to flat space only in four dimensions. Within dimensional regularization the term quadratic in the Riemann tensor should be added to the ation.

We work in the linearized theory around the flat space metric

$$
g_{\mu \nu}=\eta_{\mu v}+h_{\mu \nu}
$$

where we choose the convention $\eta_{\mu v}=\operatorname{diag}(+1,-1,-1,-1)$ in four dimensions. In $D$ dimensions $\eta_{\mu \nu} \eta^{\mu \nu}=D$. Further it is understood that indices are raised and lowered with the Minkowski metric $\eta_{\mu v}$.

Gauge transformation are generated by diffeomorphisms $x^{\mu} \rightarrow x^{\mu}+\zeta^{\mu}(x)$ and have the form

$$
h_{\mu \nu} \rightarrow h_{\mu v}+\partial_{\mu} \zeta_{v}+\partial_{\nu} \zeta_{\mu}+\left(h_{\lambda \mu} \partial_{v}+h_{\lambda v} \partial_{\mu}+\left(\partial_{\lambda} h_{\mu \nu}\right)\right) \zeta^{\lambda}
$$

here $\zeta_{\mu}(x)$ are arbitrary functions.

According to Faddeev-Popov quantization one should add to the action the gauge fixing term which we choose in the form

$$
S_{g f}=-\frac{1}{2 \xi} \int d^{D} x F_{\mu} \partial_{\nu} \partial^{v} F^{\mu}
$$

where $F^{\mu}=\partial_{v} h^{\nu \mu}, \xi$ is the gauge parameter.

One should also add the ghost term

$$
\begin{gathered}
S_{\text {ghost }}=\int d^{D} x d^{D} y \bar{C}_{\mu}(x) \frac{\delta F^{\mu}(x)}{\delta \zeta_{\nu}(y)} C_{\nu}(y)= \\
\int d^{D} x \partial^{v} \overline{C^{\mu}}\left[\partial_{\nu} C_{\mu}+\partial_{\mu} C_{v}+h_{\lambda \mu} \partial_{\nu} C^{\lambda}+h_{\lambda \nu} \partial_{\mu} C^{\lambda}+\left(\partial_{\lambda} h_{\mu v}\right) C^{\lambda}\right]
\end{gathered}
$$

where $\bar{C}$ and $C$ are ghost fields. Thus one gets the following generating functional for Green functions of gravitons

$$
Z(J)=N \int d h_{\mu \nu} d C_{\lambda} d \overline{C_{\rho}} \exp \left[i\left(S_{s y m}+S_{g f}+S_{g h o s t}+d^{D} x \mu^{-2 \epsilon} J_{\mu \nu} h^{\mu v}\right)\right],
$$

where as usual in the functional integral, $N$ is the normalization factor and $J_{\mu \nu}$ is the source of the gravitational field.

We work within perturbation theory, so we make the shift of the fields

$$
h_{\mu v} \rightarrow M_{P l} \mu^{-\epsilon} h_{\mu v}
$$


Thus perturbative expansion goes in the invers powers of the Plank mass or, in other words, in the powers of the Newton coupling constant $G$.

To derive the graviton propagator we make the Fourier transform to the momentum space and write the quadratic in $h_{\mu \nu}$ form

$$
\begin{aligned}
Q_{\mu \nu \rho \sigma}= & \frac{1}{4} \int d^{D} k h^{\mu \nu}(-k)\left[\left(k^{2}+M_{P l}^{-2} k^{4}(\alpha+4 \delta)\right) P_{\mu \nu \rho \sigma}^{(2)}\right. \\
& +k^{2}\left(-2+4 M_{P l}^{-2} k^{2}(\alpha+3 \beta+\delta)\right) P_{\mu \nu \rho \sigma}^{(0-s)} \\
& \left.+\frac{1}{\xi} M_{P l}^{-2} k^{4}\left(P_{\mu \nu \rho \sigma}^{(1)}+2 P_{\mu \nu \rho \sigma}^{(0-w)}\right)\right] h^{\rho \sigma}(k),
\end{aligned}
$$

where $P_{\mu \nu \rho \sigma}^{(i)}$ are projectors to the spin-2, spin-1 and spin-0 components of the field correspondingly:

$$
\begin{gathered}
P_{\mu \nu \rho \sigma}^{(2)}=\frac{1}{2}\left(\Theta_{\mu \rho} \Theta_{\nu \sigma}+\Theta_{\mu \sigma} \Theta_{\nu \rho}\right)-\frac{1}{3} \Theta_{\mu \nu} \Theta_{\rho \sigma}, \\
P_{\mu \nu \rho \sigma}^{(1)}=\frac{1}{2}\left(\Theta_{\mu \rho} \omega_{\nu \sigma}+\Theta_{\mu \sigma} \omega_{\nu \rho}+\Theta_{\nu \rho} \omega_{\mu \sigma}+\Theta_{\nu \sigma} \omega_{\mu \rho}\right), \\
P_{\mu \nu \rho \sigma}^{(0-s)}=\frac{1}{3} \Theta_{\mu \nu} \Theta_{\rho \sigma}, \\
P_{\mu \nu \rho \sigma}^{(0-w)}=\omega_{\mu \nu} \omega_{\rho \sigma} .
\end{gathered}
$$

Here $\Theta_{\mu v}=\eta_{\mu v}-k_{\mu} k_{v} / k^{2}$ and $\omega_{\mu v}=k_{\mu} k_{v} / k^{2}$ are the transvers and longitudinal projectors for vector quantities.

The sum of the projectors is the unity

$$
P_{\mu \nu \rho \sigma}^{(2)}+P_{\mu \nu \rho \sigma}^{(1)}+P_{\mu \nu \rho \sigma}^{(0-s)}+P_{\mu \nu \rho \sigma}^{(0-w)}=\frac{1}{2}\left(\eta_{\mu \rho} \eta_{\nu \sigma}+\eta_{\mu \sigma} \eta_{\nu \rho}\right) .
$$

Note that the expression (9) essentially differs from the analogous one in ref. [5] by the absence of $\epsilon$-dependent contributions. (9):

To obtain the graviton propagator $D_{\mu \nu \rho \sigma}$ one inverts the matrix in the square brackets of

$$
[Q]_{\mu \nu \kappa \lambda} D^{\kappa \lambda \rho \sigma}=\frac{1}{2}\left(\delta_{\mu}^{\rho} \delta_{v}^{\sigma}+\delta_{\mu}^{\sigma} \delta_{v}^{\rho}\right) .
$$

Thus we get for the propagator

$$
\begin{gathered}
D_{\mu \nu \rho \sigma}=\frac{1}{i(2 \pi)^{D}}\left[\frac{4}{k^{2}}\left(\frac{1}{1+M_{P l}^{-2} k^{2}(\alpha+4 \delta)}\right) P_{\mu \nu \rho \sigma}^{(2)}\right. \\
-\frac{2}{k^{2}}\left(\frac{1+2 \epsilon \frac{1-M_{P l}^{-2} k^{2}(\alpha+4 \beta)}{1+M_{P l}^{-2} k^{2}(\alpha+4 \delta)}}{1-\epsilon-M_{P l}^{-2} k^{2}((2 \alpha+6 \beta+2 \delta)-\epsilon(\alpha+4 \beta))}\right) P_{\mu \nu \rho \sigma}^{(0-s)} \\
\left.+4 \xi \frac{1}{M_{P l}^{-2} k^{4}}\left(P_{\mu \nu \rho \sigma}^{(1)}+\frac{1}{2} P_{\mu \nu \rho \sigma}^{(0-w)}\right)\right] .
\end{gathered}
$$

Let us perform partial fractioning. Then the graviton propagation takes the form

$$
D_{\mu \nu \rho \sigma}=\frac{1}{i(2 \pi)^{D}}\left[4 P_{\mu \nu \rho \sigma}^{(2)}\left(\frac{1}{k^{2}}-\frac{1}{k^{2}-M_{P l}^{2} /(-\alpha-4 \delta)}\right)\right.
$$




$$
\begin{gathered}
-2 \frac{P_{\mu \nu \rho \sigma}^{(0-s)}}{1-\epsilon}\left(1+2 \epsilon \frac{1-M_{P l}^{-2} k^{2}(\alpha+4 \beta)}{1+M_{P l}^{-2} k^{2}(\alpha+4 \delta)}\right) \\
\left(\frac{1}{k^{2}}-\frac{1}{k^{2}-M_{P l}^{2}(1-\epsilon) /(2 \alpha+6 \beta+2 \delta-\epsilon(\alpha+4 \beta))}\right) \\
\left.+\frac{4 \xi}{M_{P l}^{-2} k^{4}}\left(P_{\mu \nu \rho \sigma}^{(1)}+\frac{1}{2} P_{\mu \nu \rho \sigma}^{(0-w)}\right)\right] .
\end{gathered}
$$

It is interesting to note that the position of one of the poles in the term with $P_{\mu \nu \rho \sigma}^{(0-s)}$ depends on the regularization parameter $\epsilon$. The residues of both poles in this term also depend on $\epsilon$. Thus it is clear that poles and residues of the tree level propagator do not have direct physical meaning.

In the limit of four dimensions we get for the graviton propagator

$$
\begin{gathered}
D_{\mu \nu \rho \sigma}=\frac{4}{i(2 \pi)^{D}}\left[\frac{P_{\mu \nu \rho \sigma}^{(2)}-\frac{1}{2} P_{\mu \nu \rho \sigma}^{(0-s)}}{k^{2}}-\frac{P_{\mu \nu \rho \sigma}^{(2)}}{k^{2}-M_{P l}^{2} /(-\alpha-4 \delta)}\right. \\
\left.+\left(\frac{1}{2}\right) \frac{P_{\mu \nu \rho \sigma}^{(0-s)}}{k^{2}-M_{P l}^{2} /(2 \alpha+6 \beta+2 \delta)}+\frac{\xi}{M_{P l}^{-2} k^{4}}\left(P_{\mu \nu \rho \sigma}^{(1)}+\frac{1}{2} P_{\mu \nu \rho \sigma}^{(0-w)}\right)\right],
\end{gathered}
$$

Within classical four-derivative gravity for a point particle with the energy-momentum tensor $T_{\mu \nu}=\delta_{\mu}^{0} \delta_{v}^{0} M \delta^{3}(x)$ the gravitational field has the form [3]

$$
V(r)=\frac{M}{2 \pi M_{P l}^{2}}\left(-\frac{1}{4 r}+\frac{e^{-m_{2} r}}{3 r}-\frac{e^{-m_{0} r}}{12 r}\right),
$$

where in our notations $m_{2}^{2}=M_{P l}^{2} /(-\alpha-4 \delta)$ and $m_{0}^{2}=M_{P l}^{2} /(2 \alpha+6 \beta+2 \delta)$ are the squared masses of the massive spin- 2 and spin-0 gravitons correspondingly. Naturally the values of $\alpha, \beta$ and $\delta$ should be chosen to ensure positivity of the masses. As it was mentioned in [2,3] these masses can be chosen large enough to have agreements with present experiments.

The propagator (18) reproduces this expression after the calculation of the corresponding tree level Feynman diagram describing interaction of two point-like particles. The propagator presented in [2] contains some errors and dos not reproduce the expression (19).

The second term in the graviton propagator (18) has the unusual minus sign and that is why it is interpreted as the massive spin- 2 ghost. To preserve renormalizability of the quantum theory one should shift all poles in propagators in Feynman integrals in the same manner $k^{2} \rightarrow k^{2}+i 0$, hence the ghost state should be considered as the state with the negative metric [2]. This was the reason to make the statement $[2,3]$ about violation either unitarity or causality in the model.

But this massive spin-2 state is unstable since it can decay into massless gravitons. Thus it does not appear as the asymptotic state of the $S$-matrix. Correspondingly only particles with the positive metric participate in the scattering processes as external particles and unitarity is preserved in the theory.

It should be mentioned that the $S$-matrix by construction satisfies the unitarity relation

$$
S^{+} S=1
$$

in the theories with Hermitian Lagrangians, see [6].

It can be seen if one represents the $S$-matrix as the $T$-exponent within operator formalism:

$$
S=T\left(e^{i \int L(x) d x}\right)
$$


Unitarity of the $S$-matrix in the presence of negative metric states was considered in particular in $[7,8]$ where the question of causality was also studied.

It should be also mentioned that the tree level propagator (18) will be essentially modified after the summation of one-loop corrections. Because of the mignus sign in the second term of (18) the one-loop correction due to the diagram with the massless graviton in the loop will shift the pole of the ghost from the real value $k^{2}=M_{P l}^{2} /(-\alpha-4 \delta)$ to the complex value $k^{2}=M_{P l}^{2} /(-\alpha-4 \delta)-i \Gamma$, where $\Gamma$ is the decay width of the massive spin-2 graviton into the pare of massless gravitons. The complex pole is located on the unphysical Riemann sheet. This is analogous to the known virtual level in the neutron-proton system with antiparallel spins [9].

We have considered only purely gravitational action. But us it was shown in [2] one can include the matter fields in the theory straightforwardly.

\section{Conclusions}

We have demonstrated above unitarity of quantum gravity with fourth derivatives of metrics. This model is also known to be renormalizable. Thus we conclude that the considered quadratic quantum gravity is the proper candidate for fundamental quantum theory of gravitation.

\section{Acknowledgments}

The author is grateful to the Organizers of the XX-th International Seminar 'Quarks-2018' for kind hospitality and to the Participants for helpful discussions.

\section{References}

[1] G.'t Hooft and M.J.G. Veltman, Ann.Inst.H.Poincare Phys.Theor. A20 (1974) 69.

[2] K.S. Stelle, Phys. Rev. D 16 (1977) 953.

[3] K.S. Stelle, Gen. Rel. Grav. 9 (1978) 353.

[4] A.O. Barvinsky, D. Blas, M. Herrero-Valea, S.M. Sibiryakov, C.F. Steinwatchs, arXiv:1705.03480 [hep-ph].

[5] A. Salvio and A. Strumia, JHEP 1406 (2014) 080 [arXiv:1403.4226 [hep-ph]].

[6] N.N. Bogolubov and D.V. Shirkov, Introduction to the Theory of Quantized Fields, Moscow, Nauka (1976).

[7] T.D. Lee and G.C. Wick, Nucl. Phys. B 9 (1969) 209; Nucl. Phys. B 10 (1969) 1; Phys. Rev. D 2 (1970) 1033; Phys.Rev. D3 (1971) 1046.

[8] R.E. Cutcosky, P.V. Landshoff, D.I. Olive and J.C. Polkinghome, Nucl. Phys. B 12 (1969) 281.

[9] L.D. Landau and E.M. Lifshits, Quantum Mechanics : Non-Relativistic Theory, Course of Theoretical Physics, Vol.3, Pergamon Press (1991). 